TMUP-HEL-9306

June, 1993

\title{
Large-N Collective Field Theory Applied to Anyons in Magnetic Fields
}

\author{
Hideaki Hiro-Oka† and Hisakazu Minakatał \\ Department of Physics \\ Tokyo Metropolitan University \\ 1-1 Minami-Osawa Hachioji, Tokyo 192-03, Japan
}

\begin{abstract}
$\mathfrak{A b s t r a c t}$
We present a large- $N$ collective field formalism for anyons in external magnetic fields interacting with arbitrary two-body potential. We discuss how the Landau level is reproduced in our framework. We apply it to the soluble model for anyons proposed by Girvin et al., and obtain the dispersion relation of collective modes for arbitrary statistical parameters.
\end{abstract}

$\dagger$ e-mail: hiro-oka@phys.metro-u.ac.jp

$\ddagger$ e-mail: minakata@phys.metro-u.ac.jp 
The large- $N$ collective field theory [1] has been successfully applied to a wide variety of problems, the plasma oscillations, matrix models, and the fractional quantum Hall system [2,3]. In this paper we attempt to extend its applicability to the system of fractional statistics particles, anyons [4], in magnetic fields. Since this system is closely related to the quantum Hall system [5] including hierarchical excited states, it may be meaningful to develop its treatment by the collective field method.

We first extend the collective field formalism so as to include gauge fields. We believe that our method is one of the first explicit realizations of the collective field theory including gauge fields. At the end of this paper we shall make some comments on the similar approach taken in the literature [6].

We shall start by introducing our method. For definiteness we shall work with the following simple $N$-body Hamiltonian with arbitrary gauge fields $A$ in $2+1$ dimensions

$$
H=\frac{1}{2} \sum_{a=1}^{N}\left(-i \partial_{a}+A\left(x_{a}\right)\right)^{2}
$$

with the gauge condition $\partial A=0$. The mass $m$ and the coupling constant $e$ are set to unities throughout this paper to simplfy our equations. The convensional way of treating this system is to solve the many-body Schrödinger equation

$$
H \psi\left(x_{1}, \cdots, x_{N}\right)=E \psi\left(x_{1}, \cdots, x_{N}\right) .
$$

We treat this system by using the large- $N$ collective field formalism. Following by now familiar method we shall rewrite above Hamiltonian into the effective Hamiltonian in terms of collective variables. We introduce a collective variable $Q^{a}$ by defining the point canonical transformation

$$
x^{a} \longrightarrow Q^{a}=f^{a}(x),
$$

and its inverse transformation

$$
x^{a}=F^{a}(Q) .
$$

By the chain rule of differentiation the Hamiltonian (1) is written as

$$
H=\frac{1}{2} \sum_{a=1}^{N}\left(i \omega^{a} P_{a}+\sum_{b=1}^{N} \Omega^{a b} P_{a} P_{b}-\gamma^{a} P_{a} \tilde{A}-2 \tilde{A} \gamma^{a} P_{a}+\tilde{A}^{2}\right),
$$

where

$$
\begin{gathered}
\omega^{a}=-\sum_{b=1}^{N} \frac{\partial^{2} f^{a}}{\partial x^{b^{2}}}, \quad P_{a}=\frac{\partial}{i \partial Q^{a}}, \quad \Omega^{a b}=\sum_{c=1}^{N} \frac{\partial f^{a}}{\partial x^{c}} \frac{\partial f^{b}}{\partial x^{c}} \\
-2-
\end{gathered}
$$




$$
\gamma^{a}=\sum_{b=1}^{N} \frac{\partial Q^{a}}{\partial x^{b}}, \quad \tilde{A}(Q)=A(F(Q))
$$

This expression (5) itself is not hermitian under the following naïve hermitian conjugation

$$
P_{a}^{\dagger}=P_{a}, \quad Q^{a \dagger}=Q^{a} .
$$

As is well understood this does not mean any trouble. The Hamiltonian which is hermitian in the functional space of $\sqrt{J} \psi$ is the effective Hamiltonian

$$
H_{e f f}=J^{1 / 2} H J^{-1 / 2}
$$

where $J$ is the Jacobian of the transformation (3). We define a new variable $C$ as

$$
\begin{aligned}
J^{1 / 2} P_{a} J^{-1 / 2} & =P_{a}+i C_{a}, \\
C_{a} & =\frac{1}{2} \frac{\partial}{\partial Q} \ln J,
\end{aligned}
$$

in order to bypass the difficulty of evaluating Jacobian. Substituting (10) into the expression (9) and imposing the hermiticity condition $H_{\text {eff }}^{\dagger}-H_{\text {eff }}=0$, we obtain the following equation for $C$

$$
\begin{aligned}
& \sum_{a=1}^{N} \frac{\partial}{\partial Q^{a}}\left\{\omega^{a}+\sum_{b=1}^{N}\left(2 \Omega^{a b} C_{b}+\frac{\partial \Omega^{a b}}{\partial Q^{b}}\right)\right\} \\
& \quad+i \sum_{a=1}^{N}\left[2\left\{\omega^{a}+\sum_{b=1}^{N}\left(2 \Omega^{a b} C_{b}+\frac{\partial \Omega^{a b}}{\partial Q^{b}}\right)\right\} P_{a}+2 \tilde{A}\left(2 \gamma^{a} C_{a}-\frac{\partial \gamma^{a}}{\partial Q^{a}}\right)\right]=0 .
\end{aligned}
$$

The real and imaginary parts of left hand side in (11) should vanish independently. Then one might worry that $C_{a}$ is overdetermined. But it is not the case. One can show that the solution of the equation

$$
\omega^{a}+\sum_{b=1}^{N}\left(2 \Omega^{a b} C_{b}+\frac{\partial \Omega^{a b}}{\partial Q^{b}}\right)=0
$$

simultaneously solves the equation of vanishing imaginary part in the case of the transformation

$$
\phi(x)=\frac{1}{N} \sum_{i=1}^{N} \delta\left(x-x_{i}\right), \quad \phi_{k}=\int d x e^{-i k x} \phi(x) .
$$

We will describe a short proof of this statement in Appendix. We note that this feature should be generically true for any choice of the collective variable 
besides (13). If it were not for the case we would have to make an ingenious choice of the gauge fields so that the effective Hamiltonian becomes hermitian.

We utilize the density variable $\phi$ in (13) as an appropriate collective variable to discuss the low energy collective excitation of the system. Since we only need to solve (12) and it is the same equation as that without gauge fields we can just go straight ahead as in Ref.2.

Solving (12) for $C$ and putting it into (9) we can obtain a complete effective Hamiltonian up to a total divergence term. The Hamiltonian in terms of the collective variable $\phi$ and its conjugate momentum $\pi$ is finally written as

$$
H_{e f f}=\frac{1}{2} \int d x\left(\frac{\partial \pi}{N}+A\right) N \phi\left(\frac{\partial \pi}{N}+A\right)+\frac{N}{8} \int d x \frac{(\partial \phi)^{2}}{\phi},
$$

The conjugate variable $\pi$ is defined as $\pi_{k}=\frac{1}{i} \frac{\partial}{\partial \phi_{-k}}$ in momentum space and satisfies the commutation relation

$$
\left[\phi(x), \pi\left(x^{\prime}\right)\right]=i \delta\left(x-x^{\prime}\right)
$$

in the large $N$ limit. In this way we have obtained the effective Hamiltonian with gauge field expressed by the collective variables.

In the following we analyze the collective motion of anyons by means of the collective field formalism just developed. Instead of working with ideal anyon Hamiltonian (1) with Schrödinger's wave function obeying fractional statistics we make a singular gauge transformation to have an interacting boson representation of the ideal anyon gas. The Hamiltonian is given as

$$
H=\sum_{a=1}^{N} \frac{1}{2}\left(-i \partial_{a}+a\left(x_{a}\right)\right)^{2}
$$

where $a$ is the so-called fictitious gauge field written as

$$
a_{i}\left(x_{a}\right)=\frac{\theta}{\pi} \sum_{b \neq a}^{N} \frac{\epsilon_{i j}\left(x_{a}-x_{b}\right)^{j}}{\left|x_{a}-x_{b}\right|^{2}}, \quad \theta \in[0, \pi],
$$

where the spatial index $i$ is explicitly indicated. The parameter $\theta$ interpolates the two special limits, $\theta \rightarrow 0$ (boson) and $\theta \rightarrow \pi$ (fermion).

We consider, for later convenience, more generic case of anyons in real (opposed to fictitious) magnetic field interacting with each other by the potential $U$. The Hamiltonian is given by

$$
H=\sum_{a=1}^{N} \frac{1}{2}\left(-i \partial_{a}+a\left(x_{a}\right)+A\left(x_{a}\right)\right)^{2}+\sum_{a<b}^{N} U\left(x_{a}-x_{b}\right)
$$


where $A$ is the real gauge field yielding the external constant magnetic field $B$ by $\nabla \times A=B$, and $U$ is the two body interaction whose explicit form will be specified later.

Following the same procedure as before it is easy to derive the effective Hamiltonian corresponding to that of (18) to the following,

$$
\begin{aligned}
H= & \frac{1}{2} \int d x\left(\frac{\partial \pi}{N}+a+A\right) N \phi\left(\frac{\partial \pi}{N}+a+A\right)+\frac{N}{8} \int d x \frac{(\partial \phi)^{2}}{\phi} \\
& +\frac{N^{2}}{2} \int d x d y\left(\phi-\frac{1}{V}\right) U(x-y)\left(\phi-\frac{1}{V}\right)+\lambda\left(\int d x \phi-1\right) .
\end{aligned}
$$

where

$$
a_{i}(x)=\frac{\theta}{\pi} N \int d y \frac{\epsilon_{i j}(x-y)^{j}}{|x-y|^{2}} \phi(y) .
$$

is the corresponding expression of (17) in terms of the density variable. $V$ is the volume of the system. The last term in (19) is due to the constraint $\int d x \phi=1$ and $\lambda$ is the Lagrange multiplier (chemical potential).

The ground state of this system can be given by solving the following equations

$$
\begin{gathered}
\nabla\left\{N \phi\left(\frac{\nabla \pi}{N}+a+A\right)\right\}=0 \\
-\frac{N}{8}\left(\frac{\nabla \phi}{\phi}\right)^{2}-\frac{N}{4} \nabla\left(\frac{\nabla \phi}{\phi}\right)+\frac{\theta}{\pi} N^{2} \int d y \phi \nabla \ln |x-y| \times\left(\frac{\nabla \pi}{N}+a+A\right) \\
+\frac{N}{2}\left(\frac{\nabla \pi}{N}+a+A\right)^{2}+\frac{N^{2}}{2} \int d y U(x-y)\left(\phi(y)-\frac{1}{V}\right)-\lambda=0
\end{gathered}
$$

Assuming that the ground state has no vortex and that it is invariant under the translation, the solution can be given as

$$
\phi_{0}=\frac{1}{V} \text {. }
$$

Moreover, we notice that

$$
\frac{\nabla \pi_{0}}{N}+a_{0}+A=0
$$

satisfies these equations (21) and (22) under the solution (23). Here $a_{0}$ is the mean field defined by

$$
a_{i}(x)=a_{0 i}(x)+\frac{\theta}{\pi} N \int d y \frac{\epsilon_{i j}(x-y)^{j}}{|x-y|^{2}}\left(\phi(y)-\frac{1}{V}\right) .
$$


As a solution of the equation (24) we take

$$
\pi_{0}=0
$$

and

$$
a_{0}+A=0 .
$$

The latter equation means that the fictitious megnetic field organizes itself so as to cancell the applied real magnetic field. If the magnetic fields would not cancell with each other, we would have to have a singularity in $\pi$ variable. This is easily seen by (24) which states that $\nabla \times\left(\nabla \pi_{0}\right) \neq 0$ unless $\nabla \times\left(a_{0}+A\right)=0$. The singularity of $\pi$ variable implies a real vortex. Therefore, our solution to (24) means the absense of extra real vortices.

The validity of taking the above solution to (24) can be explicitly checked. The second equation (27) implies that the ground state value $\phi_{0}$ is determined by the external magnetic field $B$. That is, the ground state density $\rho_{g}$ is given as

$$
\rho_{g}=\frac{B}{2 \theta}
$$

For fermions $(\theta=\pi)$ this gives $\rho_{g}=B / 2 \pi$, the well-known result for the degeneracy of the lowest Landau level. Therefore, our solution (27) allows a very simple interpretation of completely filled lowest Landau level in this case. It is amusing to observe that the density (28) interporates between the Landau level degeneracy for the fermion case $(\theta=\pi)$ and the Bose condensate for the bosonic case $(\theta \rightarrow 0)$.

Before moving to the analysis of collective excitations let us make a short comment on ideal anyon gas without external magnetic field. In the absence of $A$ in (24) we have to deal with the singular configuration of the $\pi$ field, the Chern-Simons vortex. In this case the $\phi$ field must vanish at the location of vortices and we are not allowed to have a constant solution (23). Therefore the treatment of the ideal anyon gas is far from straightforward in the large- $N$ collective field theory. We hope to return to it in the future.

We analyze collective excitations as small oscillations around the ground state given by (26) and (27). We expand the $\phi$ and the $\pi$ variables around their ground state values,

$$
\phi=\phi_{0}+\frac{1}{\sqrt{N}} \eta, \quad \pi=\pi_{0}+\sqrt{N} \xi
$$

The prefactors of the fluctuation $1 / \sqrt{N}$ and $\sqrt{N}$ are determined in consistent with the large- $N$ expansion and the canonical commutation relation. As a mode decomposition we expand $\eta$ and $\xi$ by using the plane wave, i.e.

$$
\begin{gathered}
\eta=\frac{1}{V} \sum_{k \neq 0} \sqrt{k^{2}} e^{i k x} q_{k}, \\
\xi=\sum_{k \neq 0} \frac{1}{\sqrt{k^{2}}} e^{-i k x} p_{k}, \\
-6-
\end{gathered}
$$


Inserting equations (23), (26), and (30) into the Hamiltonian (19) and picking up the $O\left(N^{0}\right)$ terms, we obtain the simple harmonic oscillator type Hamiltonian

$$
H_{N^{0}}=\sum_{k \neq 0} \frac{1}{2}\left(\left|p_{k}\right|^{2}+\omega_{k}^{2}\left|q_{k}\right|^{2}\right)
$$

where

$$
\omega_{k}^{2}=\frac{k^{4}}{4}+4 \theta^{2} \rho^{2}+\rho k^{2} U_{k}
$$

and $U_{k}$ is the Fourier components of $U(x-y)$. This is the central result of our paper.

Let us make a few remarks:

(i) The first term, which dominates in short wavelength limit, $k \gg 1$, is the familiar kinetic term of non-relativistic particles. In this limit the information of the fractional statistics is lost as one can expect.

(ii) The second term, which is of order $\hbar^{0}$, dominates in the classical region (or in the long wavelength limit). This is the gap that can be interpreted as the Landau level interval. To see this we note that $\rho$ is given by (28) for the ground state. Then $\omega=B$ which is nothing but the cyclotron frequency under our choice of the units $m=1$ and $e / c=1$. It is also natural that the cyclotron frequency is independent of the $\theta$ parameter. Thus our formalism passes the test [7] using Kohn's theorem [8].

Let us discuss a soluble model of fractional statistics $[9,10]$ as a special example of our generic Hamiltonian (18). In this model the potential $U$ is given by

$$
U(x-y)=2 \theta \delta^{(2)}(x-y)
$$

In this case the Fourier component is given as $U_{k}=2 \theta$ and the energy level given by (32) simplifies:

$$
E_{k}=\hbar \omega_{k}=\frac{k^{2}}{2}+2 \theta \rho
$$

This result at $\theta=\pi / 2$ (semion case) is in agreement with the one derived by Girvin et al. [9] using the plasma analogy. Our result generalizes it to arbitrary $\theta$ cases.

Next we shall consider the Coulomb interaction

$$
U(x-y)=\frac{1}{\epsilon|x-y|},
$$

where $\epsilon$ is the background dielectric constant. The Fourier component $U_{k}$ is given by

$$
\begin{gathered}
U_{k}=\frac{2 \pi}{\epsilon k} . \\
-7-
\end{gathered}
$$


In this case the frequency $(32)$ becomes, in long wavelength limit $(k \ll 1)$, as

$$
\omega_{k}=B+\frac{\pi}{2 \theta \epsilon} k
$$

This agrees with the result of RPA calculation by Kallin and Halperin [11] at $\theta=\pi$ (fermion), and reproduces that of Zhang [7] for general $\theta$ cases derived by using the density-density correlation function.

A closely related but different collective field formalism of anyon system has been proposed by Andrić and Bardek [6]. While they employ the same technology as ours two formalisms differ because the starting Hamiltonians differ. Their Hamiltonian is supposed to act to the symmetric wave function constructed by removing an antisymmetrized factor. As a consequence it is non-hermitian. If we repeat the similar fluctuation analysis using their collective field Hamiltonian, eq. (16) in Ref.6, we obtain as $\omega_{k}^{2}$

$$
\omega_{k}^{2}=\omega_{k}^{2}(\text { ours })+\frac{\theta}{4 \pi}\left(\frac{\theta}{4 \pi}-1\right) k^{4}-\frac{\theta}{\pi}\left(\frac{\theta}{2 \pi}-1\right) 2 \pi \rho k^{2} .
$$

Therefore, two formalisms differ also in physical results.

To summarize: We have constructed a large- $N$ collective field theory for anyons in external magnetic fields interacting with an arbitrary two-body potential. We illuminated how the Landau level is reproduced in our framework and clarified the basic picture behind this, namely, the cancellation between the real and the fictitious magnetic fields. We applied our collective field formalism to uncover the low energy collective excitations around the ground state. In particular we obtained the dispersion relation for the collective mode in the soluble model of anyons for generic value of the statistical parameter $\theta$.

\section{Acknowledgments}

The authors thank Bunji Sakita for numerous discussions on the collective field theory and for the hospitality accorded to them during a visit to City College of New York. 


\section{Appendix}

In this Appendix we show that the left hand side of (11) vanishes under the choice of the collective variable (13) when (12) is satisfied. Namely, we shall show that

$$
2 \tilde{A}\left(2 \gamma^{a} C_{a}-\frac{\partial \gamma^{a}}{\partial Q^{a}}\right)
$$

vanishes under the above conditions. For convenience, we consider the Fourier component hereafter. From (12) we have

$$
C_{k}=\frac{1}{2} \sum_{k^{\prime}}\left(\Omega^{k k^{\prime}}\right)^{-1} \omega^{k^{\prime}} .
$$

We note that under the choice (13), namely $Q^{a}=\phi_{k}$,

$$
\gamma_{k}=-i k \phi_{k}
$$

and

$$
\Omega^{k k^{\prime}}=-\frac{k k^{\prime}}{N} \phi_{k+k^{\prime}} .
$$

Using (A3) one can show that the second term of (A1) is propotional to $\sum_{k} k$ and vanishes due to the symmetry $k \leftrightarrow-k$. Whereas the first term of (A1) can be written, thanks to (A3) and (A4), as

$$
\begin{aligned}
\sum_{k} 2 \gamma^{k} C_{k} & =\sum_{k k^{\prime}} i k^{\prime} \phi_{k} \phi_{k^{\prime}} \phi_{k+k^{\prime}}^{-1} \\
& =\sum_{k k^{\prime}} \int d x_{1} d x_{2} d x_{3} i k^{\prime} e^{-i k x_{1}} \phi\left(x_{1}\right) e^{-i k^{\prime} x_{2}} \phi\left(x_{2}\right) e^{i\left(k+k^{\prime}\right) x_{3}} \phi^{-1}\left(x_{3}\right) \\
& =\sum_{k k^{\prime}} \int d x_{1} d x_{2} d x_{3} \partial \phi\left(x_{1}\right) \phi\left(x_{2}\right) \phi^{-1}\left(x_{3}\right) e^{-i k\left(x_{1}-x_{3}\right)} e^{-i k^{\prime}\left(x_{2}-x_{3}\right)} \\
& =\int d x \partial \phi(x) \\
& =0
\end{aligned}
$$

unless some topologically nontrivial configurations arise. Thus we have shown that (A1) vanishes when (12) is satisfied.

\section{References}

1. A. Jevicki and B. Sakita, Nucl. Phys. B165 (1980) 511, B185 (1981) 89.

2. B. Sakita, Quantum Theory of Many-Variable Systems and Fields (World Scientific, Singapore, 1985). 
3. B. Sakita, D.-N. Sheng, and Z.-B. Su, Phys. Rev. B44 (1991) 11510.

4. For a summary and the original references on anyons, see e.g., F. Wilczek, Fractional Statistics and Anyon Superconductivity (World Scientific, Singapore, 1990).

5. R. E. Prange and S. M. Girvin (ed.), The Quantum Hall Effect, 2nd edition (Springer-Verlag, Berlin, 1989).

6. I. Andrić and V. Bardek, Mod. Phys. Lett. A7 (1992) 3276.

7. S. C. Zhang, Int. J. Mod. Phys. B6 (1992) 25.

8. W. Kohn, Phys. Rev. 123 (1961) 1242.

9. S. M. Girvin, A. H. MacDonald, M. P. A. Fisher, S.-J. Rey, and J. P. Sethna, Phys. Rev. Lett. 65 (1990) 1671.

10. M. Greiter and F. Wilczek, Nucl. Phys. B370 (1992) 577.

11. C. Kallin and B. Halperin, Phys. Rev. B30 (1984) 5655. 